\title{
Development of a Set of Chromosome Segment Substitution Lines in Pearl Millet [Pennisetum glaucum (L.) R. Br.]
}

\author{
B. Ramana Kumari, Maria A. Kolesnikova-Allen, C. Tom Hash, S. Senthilvel, T. Nepolean, \\ P.B. Kavi Kishor, Oscar Riera-Lizarazu, J.R. Witcombe, and Rakesh K. Srivastava
}

\begin{abstract}
The detection of minor quantitative trait loci (QTL) with conventional mapping populations can be complicated by the overshadowing effect of major QTL as well as by interactions between QTL. To overcome these constraints, we developed a set of chromosome segment substitution lines (CSSLs) by introgression of overlapping chromosome segments from 863B into ICMB 841 background for use in QTL detection, fine mapping, and trait mechanism studies, especially for complex traits. Since each CSSL carries one or a few donor segments in the genetic background of the recurrent genotype, the QTL interaction is confined to genes present on small homozygous substituted segments. Advanced generation backcross progenies (1492), expected to provide coverage across the mapped length of each of the seven pearl millet linkage groups (LGs), were genotyped at 74 marker loci [(48 simple sequence repeats (SSRs), 21 single strand conformation polymorphism-single nucleotide polymorphism (SSCP-SNP), and 5 sequence tagged sites (STSs)] identifying 124 segment introgression homozygotes (13 for LG1, 9 for LG2, 10 for LG3, 41 for LG4, 23 for LG5, 11 for LG6, and 17 for LG7). These CSSLs consisted of 1-3 homozygous introgression segments substituted from $863 \mathrm{~B}$ in the genetic background of the recurrent parent ICMB 841 and among them, 54 represent unique lines with the donor chromosome segment averaging $100.69 \mathrm{cM}$. These CSSLs developed here provide a nearly ideal set of genetic stocks for mapping and fine mapping the multitude of traits for which their parents differ.
\end{abstract}

B. Ramana Kumari, C. Tom Hash, S. Senthilvel, T. Nepolean, Oscar R. Lizarazu, and Rakesh K. Srivastava, ICRISAT, Patancheru, Hyderabad, Telangana 502 324, India; P.B. Kavi Kishor, Osmania University, Hyderabad, Telangana 500 007, India; Maria A. Kolesnikova-Allen, Tun Abdul Razak Research Centre, Hertford, United Kingdom; J.R. Witcombe, Centre for Advanced Research in International Agricultural Development (CARIAD), Bangor Univ., Bangor, Gwynedd, United Kingdom. Received 4 Sep. 2013. *Corresponding author (r.k.srivastava@cgiar.org).

Abbreviations: BC, backcross; CSSLs, chromosome segment substitution lines; DFID, Department for International Development; DH, doubled haploid; LG, linkage group; MAS, marker-assisted selection; PCR, polymerase chain reaction; QTL, quantitative trait loci; RIL, recombinant inbred line; SSCP-SNP, single strand conformation polymorphism-single nucleotide polymorphism; SSRs, simple sequence repeats; STS, sequence tagged site.

$\mathrm{P}$ EARL Millet [Pennisetum glaucum (L.) R. Br.] is the most widely grown millet and is thought to have the greatest potential as a food and forage crop among cereals. It is a highly cross-pollinated diploid $(2 n=14) \mathrm{C}_{4}$ cereal with a genome size of approximately $2350 \mathrm{Mb}$ (Bennett et al., 2000) and cultivated for both grain and dry fodder in semi-arid and arid sub-Saharan Africa and South Asia. Its grain is most likely to be used for animal feed in the United States, Latin America, and Australia, and its use for this purpose is expanding rapidly in India. Pearl millet grain generally has a crude protein level (10.3\%) that is higher by 1 to $2 \%$ than sorghum [Sorghum bicolor (L.) Moench] grown with similar cultural practices. Pearl millet protein is deficient in several essential amino acids but averages 35\% higher lysine levels than sorghum (Rooney

Published in Crop Sci. 54:2175-2182 (2014).

doi: 10.2135/cropsci2013.09.0589

(C) Crop Science Society of America | 5585 Guilford Rd., Madison, WI 53711 USA

All rights reserved. No part of this periodical may be reproduced or transmitted in any form or by any means, electronic or mechanical, including photocopying, recording, or any information storage and retrieval system, without permission in writing from the publisher. Permission for printing and for reprinting the material contained herein has been obtained by the publisher. 
and McDonough 1987). Pearl millet grain has 5 to $6 \%$ oil and a lower proportion of the less-digestible, crosslinked prolamins and lower levels of tannins than sorghum grain (Jambunathan and Subramanian 1988), which together result in a markedly higher nutritional value of pearl millet grain compared to sorghum grain.

Over the last two decades, the application of molecular markers and genetic linkage maps has allowed the identification of QTL in all major crop species including pearl millet. However, it is still complicated to detect favorable alleles of complex traits such as drought and salinity tolerance and disease resistance in a crop relative or wild species because of often poor agronomic performance of such nonadapted germplasm. Specifically, functional genomics research of complex phenotypes must resolve the technical difficulties so as to efficiently identify QTL with large effects on specific target phenotypes, to efficiently finemap target QTL and determine candidate genes of QTL, and to efficiently determine and verify functions of candidate genes underlying these QTL. Different types of populations, including recombinant inbred lines (RIL), doubled haploids $(\mathrm{DH})$, backcross $(\mathrm{BC})$ or $\mathrm{F}_{2} / \mathrm{F}_{3}$ populations, have been extensively used for QTL mapping in pearl millet (Yadav et al., 2002, 2004; Bidinger et al., 2007; Nepolean et al., 2006). Nevertheless, the resolution of QTL identification can be limited in these mapping populations due to the overshadowing effects of major QTL on the estimation of the number and effect of independently segregating minor QTL as each line inherits more large chromosomal segments from one or the other parent. Chromosome segment substitution lines (CSSLs) are powerful alternative tools for detection and precise mapping of QTL of complex traits such as yield (Ali et al., 2010). Most importantly, the genetic interaction between donor alleles is limited to those between genes present on small homozygous substituted regions as each CSSL contains only one or a few introgressed segments of donor parent in a homozygous and common genetic background of the recurrent parent.

Introgression lines are typically developed by systematic backcrossing to introgress a small segment of chromosome from a donor parent into the genetic background of a recurrent parent. Sets of such lines are expected to differ for only small portions of the genome of the donor parent in an otherwise common genetic background of the recurrent parent. The substituted segments of all substitution lines cover the entire genome of the recurrent parent. Therefore, they constitute an exotic library, in which the entire donor genome is partitioned among several lines, each carrying a single homozygous introgressed segment. These homozygous lines are a small set (25-35) that differ from each other by at least a pair of introgressed segments. The advantage of these CSSLs is the complete integration of the introgressed segments with the stability of a certain character; hence, these lines are useful resources for genetic studies including detection and fine mapping of QTL for target traits. In particular, CSSLs permit the detection of QTL with small additive effects that are masked by QTL with large effects in filial generation $2\left(\mathrm{~F}_{2}\right)$ and RIL populations. Being more convenient to phenotype, and probably even more effective, the nearly isogenic nature of the CSSLs provides a relative advantage over other segregating populations when rapidly implementing a pyramiding approach via crosses, marker analysis, and phenotyping. The lines fulfilling agronomic requirements can directly be used in breeding programs as potential donors of respective traits. The CSSLs enable systematic assessment of donor parent allelic effects in the recurrent parent genetic background and thus, facilitate transferring genes from one parent to the other in marker-assisted breeding. More recently, two CSSLs from rice (Oryza sativa L.) cultivars Nipponbare/Kasalath were crossed to map QTL for mesocotyl length and also study interaction among QTL (Lee et al., 2012). Similarly, CSSLs derived from rice cultivars 93-11/Nipponbare were used to identify QTL for cooked rice elongation (Yang et al., 2013).

Considering these several uses of introgression lines, work was initiated at ICRISAT to develop a set of introgression lines in pearl millet. Two agronomically elite mapping population parental lines, viz. 863B and ICMB 841, which were known to differ for important agronomic traits such as downy mildew resistance, drought and salinity tolerance, and combining ability for many grain and stover yield and quality traits, were used to develop introgression lines having overlapping segments from $863 \mathrm{~B}$ in the genetic background of ICMB 841 via marker-assisted backcrossing. The current study was proposed to further advance these backcross progenies to develop a comprehensive set of such segment introgression lines to exploit the genetic and physiological mechanism associated with several agronomic traits of interest that remain in these two parental lines.

\section{MATERIALS AND METHODS Plant Material}

Two agronomically elite inbred seed parents, ICMB 841 and 863B, were crossed to develop a segregating population for genetic linkage map construction and trait analysis. The two parents are known to produce hybrids that distinctly differ in their response to post-flowering stress. Parent 863B (Andrews and Kumar, 1996; Rai et al., 2008) was bred from the Iniadi landrace material from Togo. It was selected for this study based on its combination of agronomic elite qualities and superior combining ability for grain filling under terminal drought conditions. Parent ICMB 841 (Singh et al., 1990) is the maintainer of the female parent of several high yielding hybrids that have been widely grown in India but lack tolerance to terminal drought stress.

Before the initiation of this research project, development of the segment substitution line set had progressed to the $\mathrm{BC}_{5} \mathrm{~F}_{2} /$ $\mathrm{BC}_{6} \mathrm{~F}_{1}$ generation with support from the U.K. Department for International Development. In these generations, plants were selected for heterozygosity for the donor parent marker alleles 


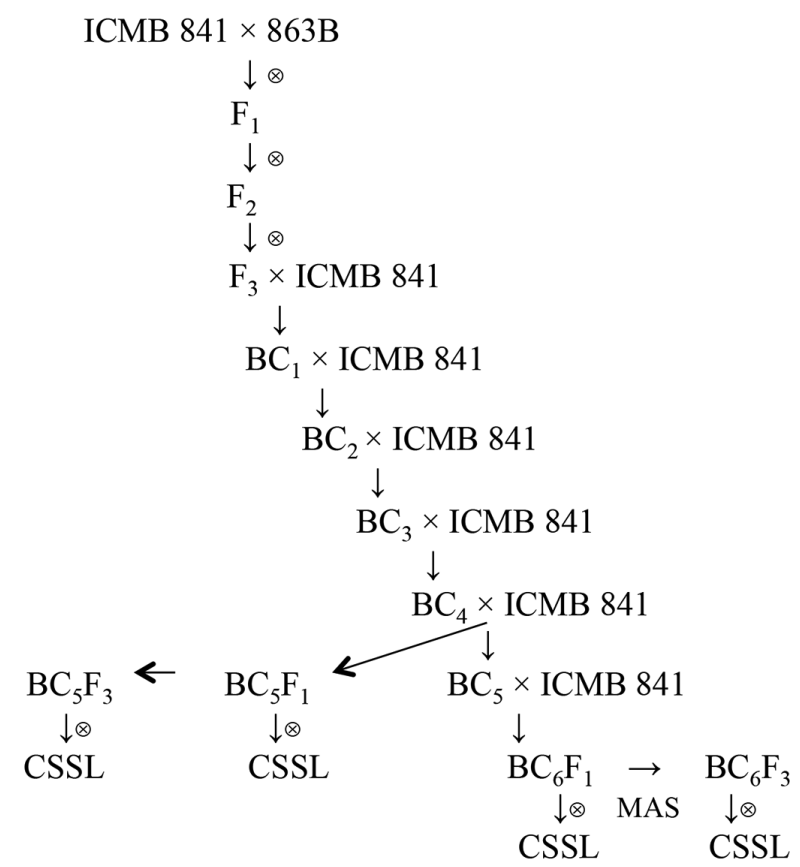

Figure 1. Flow chart of construction of chromosome segment substitution lines in pearl millet [Pennisetum glaucum (L.) R. Br.] with marker-assisted selection (MAS).

on a single linkage group (LG), or rarely two LGs, combined with homozygosity for the recurrent parent alleles across all other genomic regions for which marker data was available. A database containing marker genotype information and availability for selfed and backcrossed seed materials harvested from the $\mathrm{BC}_{5} \mathrm{~F}_{2}$ / $\mathrm{BC}_{6} \mathrm{~F}_{1}$ generation was developed and used to identify the most promising $\mathrm{BC}_{5} \mathrm{~F}_{3}$ and $\mathrm{BC}_{6} \mathrm{~F}_{2}$ families for generation of the segment introgression homozygotes. The schematic diagram for the development of CSSLs is represented in Fig. 1.

In the present study, a total of 1492 progenies (376 for LG1, 69 for LG2, 88 for LG3, 328 for LG4, 302 for LG5, 102 for LG6, and 227 for LG7) derived from the advanced backcross populations (from $\mathrm{BC}_{5} \mathrm{~F}_{3}, \mathrm{BC}_{5} \mathrm{~F}_{2}$, and $\mathrm{BC}_{6} \mathrm{~F}_{2}$ families) were selected. These plants were selected to ensure that the genetic background of CSSLs was that of the recurrent parent, ICMB 841 type, in all LGs except for one or two LGs. For genotyping, 74 polymorphic pearl millet markers were used, identified earlier by various groups: SSR (Qi et al., 2001, 2004; Allouis et al., 2001; Budak et al., 2003; Senthilvel et al., 2008), STS (Money et al., 1994), and SSCP-SNP (Bertin et al., 2005).

\section{DNA Sampling}

Genomic DNA was extracted from leaf tissue of samples of each of the selected plants, as well as their recurrent and donor parents, using a high-throughput DNA extraction protocol described by Mace et al. (2003). The quantity and quality of genomic DNA were determined by comparing the sample intensity with that of known amount of uncut $\lambda$ DNA (Amersham Biosciences) by electrophoresis on $0.8 \%$ agarose gels containing ethidium bromide $\left(0.5 \mu \mathrm{L}(10 \mathrm{~mL} \text { gel })^{-1}\right)$ and normalized to a working concentration of $2.5 \mathrm{ng} \mu \mathrm{l}^{-1}$.

\section{Genotyping}

Polymerase chain reaction (PCR) was performed in GeneAmp PCR System 9700 thermal cycler (Applied Biosystems) using 5 $\mu \mathrm{L}$ reaction volumes containing 2.5 to $5 \mathrm{ng}$ genomic DNA, $2 \mathrm{pM}$ of primer (both forward and reverse), $10 \mathrm{mM}$ of $\mathrm{MgCl}_{2}, 2 \mathrm{mM}$ dNTPs, 10X reaction buffer, and $0.1 \mathrm{U}$ Taq polymerase (AmpliTaq Gold, Applied Biosystems). A touchdown PCR amplification program was used after one denaturation step for $15 \mathrm{~min}$ at $94^{\circ} \mathrm{C}$. This program involved a denaturation step of $15 \mathrm{~s}$ at $94^{\circ} \mathrm{C}$ and an extension step of $30 \mathrm{~s}$ at $72^{\circ} \mathrm{C}$. The initial annealing step was $20 \mathrm{~s}$ at $61^{\circ} \mathrm{C}$ for one cycle, and subsequently the temperature was decreased by $1^{\circ} \mathrm{C}$ for every cycle until a final temperature of $54^{\circ} \mathrm{C}$ was achieved. The annealing temperature of $54^{\circ} \mathrm{C}$ was maintained for the last 40 cycles of amplification followed by final extension of $72^{\circ} \mathrm{C}$ for $20 \mathrm{~min}$. In case of unlabeled markers, the amplified PCR products were resolved on $6 \%$ native polyacrylamide (PAGE) gels coupled with silver staining as described by Tegelstrom (1992). In case of unlabeled STS markers and SSR markers (the forward primer was directly labeled with one of the four fluorescent dyes viz. Fam, Ned, Pet, and Vic), the amplified PCR products were resolved in capillary electrophoresis using the following procedure. Addition of a fluorescently labeled forward primer into the PCR reaction, along with reverse primers, adds the fluorescent label to the PCR amplicon. This facilitates highthroughput genotyping and marginally reduces the cost of such genotyping. Pooled PCR products of different markers labeled with different fluorescent dyes were denatured at $95^{\circ} \mathrm{C}$ for 5 min. Capillary electrophoresis of denatured pooled products was performed using a ABI3130xl DNA Genetic Analyzer (Applied Biosystems, Inc.). After completion of the electrophoresis run, the raw data files created by the ABI machine were processed through GENESCAN version 3.7 software (Applied Biosystems) for sizing the PCR amplified fragments based on their relative mobility compared to the internal Liz size standards. Allele calling was done using GENOTYPER version 3.7 software (Applied Biosystems). Based on the amplicon sizes, data were scored for all optimized primers run against template DNA from each individual sample. Data points were scored as follows: A (homozygous allele of the recurrent parent, ICMB 841), B (homozygous allele of donor parent, 863B), $\mathrm{H}$ (heterozygous presence of both parental alleles), or "-" missing data (failed amplification). Chromosome segment substitution lines were identified by the presence of homozygous segment introgression of the donor parent $863 \mathrm{~B}$ in the background of the recurrent parent ICMB 841. For each of the seven linkage groups, map length was computed by following the marker order of the pearl millet consensus map (Rajaram et. al., 2013) for the common markers. In case of unique markers from this study, map length was calculated using the best marker order suggested by MAPMAKER/EXP (Lincoln et al., 1993). Map distances were estimated in Kosambi units. Chromosome segment substitution lines were identified by the presence of homozygous segment introgression of the donor parent $863 \mathrm{~B}$ in the background of the recurrent parent ICMB 841.

\section{RESULTS}

A total of 1492 progenies derived from the advanced backcross population parents, ICMB 841 and 863B, were genotyped using 74 polymorphic markers with an average genetic 
Table 1. Summary of pearl millet [Pennisetum glaucum (L.) R. Br.] chromosome segment substitution lines (CSSLs) with 863B introgression segments in the genetic background of ICMB 841.

\begin{tabular}{|c|c|c|c|c|c|c|c|c|}
\hline Particulars & LG1 & LG2 & LG3 & LG4 & LG5 & LG6 & LG7 & Total \\
\hline No. of polymorphic markers used & 10 & 19 & 10 & 7 & 10 & 9 & 9 & 74 \\
\hline Total map length (cM) & 138.6 & 88.0 & 276.6 & 142.8 & 280.1 & 219.7 & 323.7 & 1469.5 \\
\hline Average intermarker distance (cM) & 15.4 & 4.9 & 30.7 & 23.8 & 31.1 & 27.5 & 40.5 & 173.9 \\
\hline No. of CSSLs identified out of 1492 lines & 13 & 9 & 10 & 41 & 23 & 11 & 17 & 124 \\
\hline No. of CSSLs with a single homozygous introgression segment of 863B & 5 & 9 & 5 & 33 & 9 & 9 & 15 & 85 \\
\hline No. of CSSLs with two homozygous introgression segments of $863 \mathrm{~B}$ & 8 & - & 5 & 8 & 11 & 2 & 2 & 36 \\
\hline No. of CSSLs with three introgression segments of $863 \mathrm{~B}$ & - & - & - & - & 3 & - & & 3 \\
\hline No. of CSSLs with unique introgression segments of $863 \mathrm{~B}$ & 9 & 3 & 7 & 7 & 14 & 6 & 8 & 54 \\
\hline No. of unique introgression segments of $863 \mathrm{~B}$ & 10 & 3 & 9 & 6 & 12 & 7 & 10 & 57 \\
\hline No. of recurrent parent homozygous lines identified out of 1492 lines & 143 & 13 & 4 & 62 & 43 & 8 & 25 & 298 \\
\hline No. of lines containing heterozygous segments out of 1492 lines & 220 & 47 & 74 & 225 & 236 & 83 & 185 & 1070 \\
\hline
\end{tabular}

distance of $24.83 \mathrm{cM}$ to provide coverage across most of each of the seven pearl millet linkage groups to identify chromosome segment substitution lines (CSSLs) for pearl millet. The summary of CSSLs identified is presented in Table 1.

\section{Linkage Group 1 (LG1)}

A total of $376 \mathrm{BC}_{5} \mathrm{~F}_{3}$ and $\mathrm{BC}_{6} \mathrm{~F}_{2}$ progenies (derived from 30 $\mathrm{BC}_{5} \mathrm{~F}_{2}$ and $\mathrm{BC}_{6} \mathrm{~F}_{1}$ families) were successfully genotyped with ten polymorphic markers with an average marker distance of 15.39 cM (six SSRs and four SSCP-SNPs, viz., Xpsmp2069, Xpsmp2273, Хрsmp3080, Хрsmp3017, Хрsmp3032, Xctm12, Xpsms35, Xpsms38, Xpsms58, and Xpsms86) distributed across linkage group 1 (LG1) to identify chromosome segment substitution lines for LG1. Based on the analysis of the 10 markers used, nine 863B alleles were represented in at least one plant. Based on this genotyping, 143 lines (selfed progeny of the genotyped plants) were identified as the recurrent parent homozygotes that lack any substituted donor segment from $863 \mathrm{~B}$, and 220 lines exhibited 1-3 heterozygous segments substituted from 863B. Finally, the remaining 13 lines showed 1-2 homozygous segments substituted from 863B in the genetic background of the recurrent parent ICMB 841. Among these 13 CSSLs, 5 lines have only one homozygous segment and 8 lines have two homozygous segments from the donor parent. A total of nine unique lines with 10 unique homozygous $863 \mathrm{~B}$ introgression segments were achieved with these 13 CSSLs, which subsequently constituted our CSSLs for LG1, though a few lines have overlapping segments (Fig. 2). The distribution of the introgressed segments along this chromosome was not random. The majority of $863 \mathrm{~B}$ introgression segments were often at one terminal position. The donor genome was not covered in any of these 13 CSSLS at marker Xctm12.

\section{Linkage Group 2 (LG2)}

A total of 69 individuals comprised of $\mathrm{BC}_{5} \mathrm{~F}_{3}$ and $\mathrm{BC}_{6} \mathrm{~F}_{2}$ progenies (derived from four $\mathrm{BC}_{5} \mathrm{~F}_{2}$ and $\mathrm{BC}_{6} \mathrm{~F}_{1}$ families) and 19 polymorphic markers (11 SSRs, seven SSCP-SNPs, and one STS, viz. Xpsmp2072, Xpsmp2088, Xpsmp2066,
Xpsmp2206, Xpsmp2255, Xpsmp2201, Хрsmp2231, Xpsmp2225, Xpsmp2059, Хpsmp2232, Xctm21, Xpsms13, Xpsms30, Xpsms75, Xpsms84, Xpsms82, Xpsms73, Xpsms22, and Xpsmp322) distributed across linkage group 2 (LG2) were used to identify CSSLs for LG2. The average genetic distance between markers is $4.88 \mathrm{cM}$. Based on the analysis of the 19 markers used, eight 863B alleles were represented in at least one plant. Thirteen plants were identified lacking any substituted donor segment from 863B, nine plants showed single homozygous segments substituted from the donor parent $863 \mathrm{~B}$, and the remaining 47 plants contained heterozygous donor segments. Among nine homozygous LG2 introgression lines identified, three were unique lines containing three different homozygous introgression segments of the donor parent, occupying one terminal position and part of middle portion of the LG2 (Fig. 2).

\section{Linkage Group 3 (LG3)}

To construct CSSLs for linkage group 3 (LG3), $88 \mathrm{BC}_{5} \mathrm{~F}_{3}$ progenies (derived from $8 \mathrm{BC}_{5} \mathrm{~F}_{2}$ families) were selected and genotyped with 10 polymorphic markers with an average interval of $30.71 \mathrm{cM}$ (six SSRs, three SSCP-SNPs, and one STS, viz. Xpsmp2267, Хpsmp2070, Хpsmp2214, Хpsmp2227, Xpsmp2249, Xctm10, Xpsms31, Xpsms60, Xpsms61, and Xpsmp108). Based on the analysis of the 10 markers used, eight $863 \mathrm{~B}$ alleles were represented in at least one plant. Of the 88 lines analysed, four lines had no detectable alleles from the donor parent 863B and, hence were recurrent parent allele homozygotes at all genotyped loci. Finally, 10 CSSLs were identified. Five CSSLs out of 10 showed a single homozygous donor parent introgression segment and five lines showed two homozygous introgression segments from the donor parent $863 \mathrm{~B}$ in the background of the recurrent parent ICMB 841. A total of nine unique homozygous $863 \mathrm{~B}$ introgression segments were achieved from seven unique CSSLs, which subsequently constituted our CSSLs for LG3 (Fig. 2). The distribution of the introgression segments along the chromosome was not random and could not cover the portion of LG3 that includes marker positions Xpsms61 and Xpsms31. 


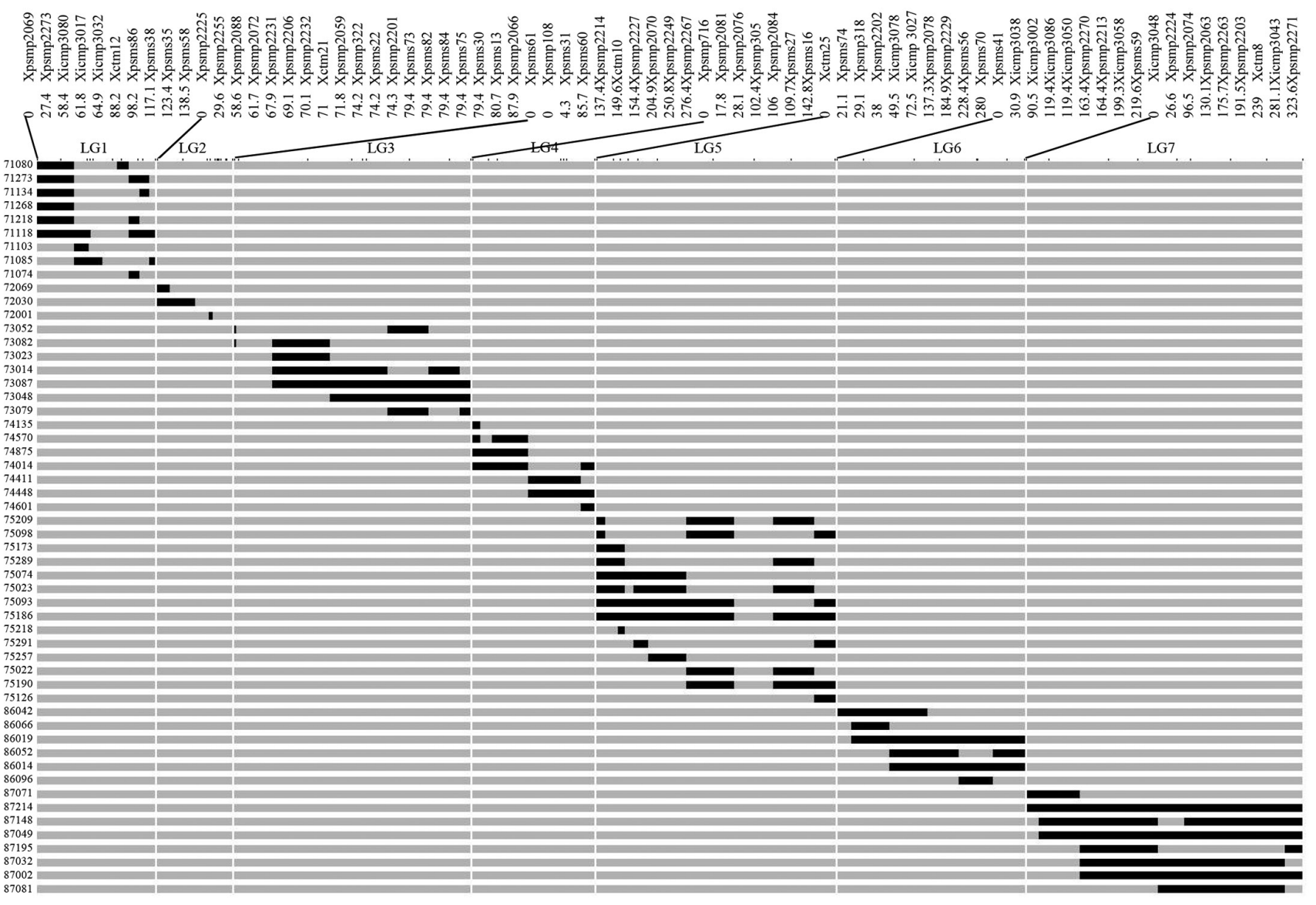

Figure 2. Graphical representation of genotype of chromosome segment substitution lines (CSSL) developed for all seven chromosomes of pearl millet [Pennisetum glaucum (L.) R. Br.]. CSSL no. and marker name with genetic distance (cM) are indicated on left side and upper side of genotypes, respectively. Black bar segments are homozygous for the donor parent, 863B alleles. Grey bar segments are homozygous for the recurrent parent, ICMB 841 alleles.

\section{Linkage Group 4 (LG4)}

A total of $328 \mathrm{BC}_{5} \mathrm{~F}_{3}, \mathrm{BC}_{5} \mathrm{~F}_{2}$, and $\mathrm{BC}_{4} \mathrm{~F}_{3}$ progenies (derived from $21 \mathrm{BC}_{5} \mathrm{~F}_{2}$, one $\mathrm{BC}_{5} \mathrm{~F}_{1}$, and four $\mathrm{BC}_{4} \mathrm{~F}_{2}$ families) were successfully genotyped with seven polymorphic markers (three SSRs, two SSCP-SNPs, and two STSs, viz. Xpsmp2081, Xpsmp2076, Xpsmp2084, Xpsms27, Xpsms16, Xpsmp 716, and Xpsmp305) distributed across the linkage group 4 (LG4) with an average marker distance of 23.8 cM to identify chromosome segment substitution lines for LG4. Based on analysis of the seven markers used, all seven alleles of 863B were represented in at least one plant. Based on this genotyping, 62 lines were identified lacking a substituted donor segment from 863B, 225 lines exhibited 1 to 4 heterozygous segments substituted from $863 \mathrm{~B}$, and 41 lines showed 1 to 2 homozygous segments substituted from $863 \mathrm{~B}$ in the background of the recurrent parent ICMB 841. Many of these lines have overlapping introgressed segments. Among these 41 lines, 33 lines showed one and 8 lines showed two homozygous introgression segments from the donor parent with the remaining genetic background of the recurrent parent. A total of six unique homozygous 863B introgression segments were achieved from seven unique CSSLs across these 41 CSSLs, which subsequently constituted our CSSLs for LG4 (Fig. 2). The distribution of the introgression segments along this chromosome was also random, overlapping in some lines and covering the majority of the both terminal portions of LG4.

\section{Linkage Group 5 (LG5)}

The CSSLs for LG5 were constructed by genotyping 302 $\mathrm{BC}_{5} \mathrm{~F}_{3}$ progenies (derived from $21 \mathrm{BC}_{5} \mathrm{~F}_{2}$ families) with 10 polymorphic markers (six SSRs, three SSCP-SNPs, and one STS viz. Xpsmp2202, Xpsmp2078, Xpsmp2229.2, Xicmp3027, Xicmp3078, Xctm25, Xpsms74, Xpsms56, $X p s m s 70$, and Xpsmp318) distributed across linkage group 5 (LG5). The marker distance ranged from 8 to $64.8 \mathrm{cM}$. Based on the analysis of the 10 markers used, all these 10 alleles of $863 \mathrm{~B}$ were represented in at least one line. Of the 302 lines (selfed progeny of the genotyped plants) analyzed, 43 lines were identified as the recurrent parent homozygotes that lack any detectable donor parent $863 \mathrm{~B}$ specific allelic pattern, and 236 lines exhibited 1 to 4 heterozygous segments substituted from 863B. Finally, 23 CSSLs were identified containing 1-3 homozygous introgression segments of the donor parent $863 \mathrm{~B}$ in the background of the recurrent parent ICMB 841 genome. Among these 23 
lines, 9 lines showed one, 11 lines showed two, and three lines showed three homozygous introgression segments from the donor parent with the remaining genetic background of the recurrent parent. A total of 12 unique homozygous $863 \mathrm{~B}$ introgression segments were achieved from 23 CSSLs, which subsequently constituted our 14 unique CSSLs for LG5 (Fig. 2). The distribution of the introgression segments could cover the entire length of LG5, except at the Xpsmp2229.1 marker locus.

\section{Linkage Group 6 (LG6)}

A total of $102 \mathrm{BC}_{5} \mathrm{~F}_{3}$ progenies (derived from $8 \mathrm{BC}_{5} \mathrm{~F}_{2}$ families) and nine polymorphic markers (seven SSRs and two SSCP-SNPs viz. Xpsmp2270, Xpsmp2213, Xicmp3002, Xicmp3058, Xicmp3086, Xicmp3050, Xicmp3038, Xpsms41, and Xpsms59) covering across linkage group 6 (LG6) (with an average marker distance of $27.45 \mathrm{cM}$ ) were successfully used to identify CSSLs for LG6. Based on the analysis of the nine markers used, all nine alleles of $863 \mathrm{~B}$ were represented in at least one line. Eight lines were identified lacking substituted donor segment from 863B, 83 lines contained heterozygous segments, and the remaining 11 lines showed single homozygous segments of $863 \mathrm{~B}$ in the genetic background of ICMB 841. Seven unique homozygous introgression segments of the donor parent were achieved from six unique CSSLs out the above set of 11 CSSLs for LG6 (Fig. 2).

\section{Linkage Group 7 (LG7)}

A total of $227 \mathrm{BC}_{6} \mathrm{~F}_{2}$ progenies (derived from two $\mathrm{BC}_{6} \mathrm{~F}_{1}$ families) were successfully genotyped with nine polymorphic SSR markers (viz. Xpsmp2224, Xpsmp2271, Xpsmp2074, Xpsmp2063, Хpsmp2263, Хрsmp2203, Xicmp3043, Xicmp3048, and Xctm8) distributed across linkage group 7 (LG7) with the distance between markers ranging from 15.8 to $69.9 \mathrm{cM}$. Based on the analysis, $863 \mathrm{~B}$ alleles for all nine markers used were represented in at least one line. Based on this genotyping results, 25 lines were identified as the recurrent parent homozygous lines, lacking any substituted donor segment from 863B, 185 lines exhibited 1-3 heterozygous segments substituted from 863B, and the remaining 17 lines showed 1-3 homozygous segments substituted from $863 \mathrm{~B}$ in the genetic background of the recurrent parent ICMB 841. Out of these 17 CSSLs, 15 lines have one homozygous introgression segment and two lines have two homozygous introgression segments of the donor parent. A total of 10 unique homozygous $863 \mathrm{~B}$ introgression segments were achieved from this set of 17 CSSLs, which subsequently constituted our eight unique CSSLs for LG7 (Fig. 2). The distribution of the introgression segments along the chromosome was not random and covered the entire length of LG7, though there were few lines having overlapping segments.

\section{DISCUSSION}

Introgression lines have been developed for various crops such as tomato (Solanum lycopersicum L.), lettuce (Latuca sativa L.), barley (Hordeum vulgare L.), rice (Oryza sativa L.), and Brassica oleracea L. During development of CSSLs, molecular marker-assisted selection (MAS) can accelerate the process. In most cases, MAS was conducted from early backcross generation as in tomato (Eshed and Zamir 1994), Brassica oleracea L. (Ramsay et al., 1996), and Brassica napus L. (Howell et al., 1996). Liu et al. (2006) delayed MAS selection until the late backcross generations during the development of 82 introgression lines from 97 selected lines of $\mathrm{BC}_{4} \mathrm{~F}_{3}$ using a synthetic wheat (Triticum aestivum L.) as the donor parent. Their results proved that marker detection at the end of the process of introgression lines development might also be efficient. Development of an incomplete set of CSSLs was reported by Kubo et al. (2002) in chromosome substitution series derived from a japonica and indica cross of rice and also by Tian et al. (2006) in construction of introgression lines carrying wild rice (Oryza rufipogon) segments in the background of indica cultivar, Guichao 2 . The latter group reported that only $67.5 \%$ of the genome coverage of Oryza rufipogon was achieved, which might be due to random selection of lines during MAS.

Usually there are two methods to develop a CSSL population. The first method involves crossing of two elite parents with subsequent backcrossing with the recurrent parent to generate $\mathrm{BC}_{3}$ individuals. Then, extensive MAS is performed until segment substitution lines overlapping the entire genome of the donor parent are developed. This method was used by Hao et al. (2006) to develop CSSLs that carried overlapping chromosome segments of the entire rice genome. Zhang et al. (2011) also developed a population of 57 CSSLs having 95 substituted segments of indica 9311, with an average of about 2.5 segments per CSSL, and eight segments per chromosome with a background of Nipponbare using marker-assisted backcrossing and high-throughput resequencing strategy. A large population containing 128 CSSLs in rice was successfully developed using a high quality physical map of ultrahigh density SNPs based on whole genome resequencing data by Xu et al. (2010). They reported more accuracy in this map than in a comparison map constructed using PCR-based markers. The second method involves choosing a specific RIL and backcrossing with the donor parent, assisted by molecular markers. Kubo et al. (1999) implemented this second approach to generate a series of indica CSSLs in the japonica background in rice.

In the present study, marker-assisted backcrossing was used to develop a set of CSSLs for pearl millet. A total of 1492 progenies (derived from $\mathrm{BC}_{5} \mathrm{~F}_{3}, \mathrm{BC}_{5} \mathrm{~F}_{2}$, and $\mathrm{BC}_{6} \mathrm{~F}_{1}$ families), expected to provide coverage across most of each of the seven pearl millet linkage groups, were selected for genotyping at 74 polymorphic marker loci to develop CSSLs for pearl millet. For all seven linkage groups of 
pearl millet, a total 124 CSSLs were detected, and among them, 54 lines were unique with one or two chromosome segments of $863 \mathrm{~B}$. These lines were consequently selected to construct a CSSL population with an average length of $100.69 \mathrm{cM}$ (6.85\% of the whole pearl millet genome) of the donor chromosome segment. These lines offer broad coverage of the whole donor genome of $863 \mathrm{~B}$ part by part in the genetic background of the recurrent parent ICMB 841. In this set of CSSLs, a few lines have overlapping segments, and all lines together cover 60 out of 74 markers used for genotyping and had alleles of the donor parent 863B. Most introgression segments were detected by one to four marker loci, with some being detected by more than four marker loci. Different introgression frequencies were observed among the seven linkage groups. Among 54 unique introgression segments, 13 introgression segments were found on LG5 but only three on LG2. However, in spite of having the largest number of polymorphic markers used for genotyping, the major portion of LG2 was not covered. Also, three regions on LG1, LG3, and LG5 were not covered by overlapping introgression segments. The low introgression ratio of the donor-recurrent parent genome may be caused by two reasons. The first one could be due to the small size of the population genotyped. For example, in the present study, only 69 plants for LG2 were selected for genotyping, and this might be the reason why only three introgression segments were detected. If more plants were screened for genotyping, more distinct introgression segments could be obtained. The second reason may be attributed to selective segregation distortion known to be present in the segregating generations of this cross.

In the present research, the complete set of CSSLs that cover the pearl millet genome could not be developed, which may impact subsequent work and QTL mapping, in particular, because some lines lack introgression segments from the donor parent. The reason could be due to the limited number of markers used and their uneven distributions across chromosomes. Only 74 markers with an average of 10.5 markers per chromosome with an average marker distance of $24.83 \mathrm{cM}$ were used. Some markers were clustered in small regions, while no polymorphic markers were found in other regions. For example, in the case of LG2, some markers were clustered at the same region with the marker distance less than $2 \mathrm{cM}$. In the case of LG3, the maximum distance between the markers observed was $81.4 \mathrm{cM}$. This could prevent the detection of some introgression segments in this area due to no marker coverage. Out of 1492 lines genotyped, 298 lines were found to be homozygous recurrent parent type and 1070 lines contained one to five heterozygous segments. The reason for obtaining recurrent parent homozygotes might be that some introgression segments of the donor parent were completely replaced by the recurrent parent during backcrossing.
The present study could be extended to complete the construction of whole genome introgression lines of the donor parent $863 \mathrm{~B}$ in the genetic background of the recurrent parent ICMB 841 by screening more $\mathrm{BC}_{5} \mathrm{~F}_{3}$ plants and by using more molecular markers. We can improve the set of CSSLs by selfing of plants with heterozygous alleles, for example from $\mathrm{BC}_{5} \mathrm{~F}_{2}$ to $\mathrm{BC}_{5} \mathrm{~F}_{3}$. Chromosome segment substitution lines are valuable because they decrease the interaction effects between QTL anchored in different segments and subsequently divide the QTL into single Mendelian factors, resulting in higher precision QTL mapping. Hence, CSSLs are an ideal novel population for QTL mapping. While densely and evenly spaced markers would be necessary for producing CSSLs with a very fine resolution, the present study has produced a useful genomic resource using a limited set of markers.

Chromosome segment substitution lines reported in the present study will provide potent tools for discovery and functional study of essential QTL and alleles in pearl millet. These will be useful in enhancing the accuracy and stability of QTL, since the conventional bi-parental mapping populations suffer from segregation distortion. Chromosome segment substitution lines may provide a platform for research on QTL fine mapping, map-based QTL cloning, and interaction effects between QTL, especially for complex traits. Ultimately, CSSLs provide an abundant germplasm for MAB to develop new cultivars with good grain and stover yield.

\section{CONCLUSIONS}

In the present study, a set of 54 chromosome segment substitution lines for pearl millet with chromosome segments of $863 \mathrm{~B}$ in the genetic background of ICMB 841 were developed for all seven LGs. For LG2, chromosome segment introgression coverage could be improved (from the present three lines) by selfing advanced backcross entries followed by genotyping with a greater number of evenly spaced polymorphic markers. We also need to generate CSSLs with smaller intervals of overlapping chromosomal introgressions with help of appropriate markers for precision studies. Early beneficiaries of this research would include public- and private sector pearl millet breeders, geneticists, and physiologists globally who are attempting to exploit molecular markers to improve biotic and abiotic stress resistance in pearl millet.

\section{Acknowledgments}

The first author gratefully acknowledges grant support from the Department of Biotechnology (DBT), Government of India. This document is an output from a series of Plant Sciences Research Programme projects funded by the U.K. Department for International Development (DFID) and administered by CAZS Natural Resources for the benefit of developing countries. The views expressed are not necessarily those of DFID. This work has been published as part of the CGIAR Research Program on Dryland Cereals. 


\section{References}

Ali, M.L., P.L. Sanchez, S. Yu, M. Lorieux, and G.C. Eizenga. 2010. Chromosome segment substitution lines: A powerful tool for the introgression of valuable genes from Oryza wild species into cultivated rice $(O$. sativa). Rice 3:218-234. doi:10.1007/s12284-010-9058-3

Allouis, S., X. Qi, S. Lindup, M.D. Gale, and K.M. Devos. 2001. Construction of a BAC library of pearl millet, Pennisetum glaucum. Theor. Appl. Genet. 102:1200-1205. doi:10.1007/s001220100559

Andrews, D.J., and K.A. Kumar. 1996. Use of the West African pearl millet landrace Iniadi in cultivar development. Plant Genet. Resour. Newsl. 105:15-22.

Bennett, M.D., P. Bhandol, and I.J. Leitch. 2000. Nuclear DNA amounts in angiosperms and their modern uses 807 new estimates. Ann. Bot. (Lond.) 86:859-909. doi:10.1006/anbo.2000.1253

Bertin, I., J.H. Zhu, and M.D. Gale. 2005. SSCP-SNP in pearl milleta new marker system for comparative genetics. Theor. Appl. Genet. 110:1467-1472. doi:10.1007/s00122-005-1981-0

Bidinger, F.R., T. Nepolean, C.T. Hash, R.S. Yadav, and C.J. Howarth. 2007. Quantitative trait loci for grain yield in pearl millet under variable postflowering moisture conditions. Crop Sci. 47:969-980. doi:10.2135/ cropsci2006.07.0465

Budak, H., F. Pedraza, P.B. Cregan, P.S. Baenzinger, and I. Dweikat. 2003. Development and utilization of SSRs to estimate the degree of genetic relationships in a collection of pearl millet germplasm. Crop Sci. 43:2284-2290. doi:10.2135/cropsci2003.2284

Eshed, Y., and D. Zamir. 1994. Introgressions from Lycopersicon pennellii can improve the soluble solids yield of tomato hybrids. Theor. Appl. Genet. 88:891-897. doi:10.1007/BF01254002

Hao, W., J. Jin, S.Y. Sun, M.Z. Zhu, and H.X. Lin. 2006. Construction of chromosome segment substitution lines carrying overlapping chromosome segments of the whole wild rice genome and identification of quantitative trait loci for rice quality. J. Plant Physiol. Mol. Biol. (In Chinese, with English abstract). 32:354-362.

Howell, P.M., D.F. Marshall, and D.J. Lydiate. 1996. Towards developing intervarietal introgression lines in Brassica napus using marker-assisted selection. Genome 39:348-358. doi:10.1139/g96-045

Jambunathan, R., and V. Subramanian. 1988. Grain quality and utilization in sorghum and pearl millet. In: J.M.J. de Wet and T.A. Preston, editors, Proceedings of the Workshop on Biotechnology for Tropical Crop Improvement, ICRISAT, Patancheru, Andhra Pradesh, India. 12-15 Jan 1987. ICRISAT Center, India. p. 133-139.

Kubo, T., K. Nakamura, and A. Yoshimura. 1999. Development of a series of Indica chromosome segment substitution lines in japonica background of rice. Rice Genet. Newsl. 16:104-106.

Kubo, T., Y. Aida, K. Nakamura, H. Tsunematsu, K. Doi, and A. Yoshimura. 2002. Reciprocal chromosome segment substitution series derived from Japonica and indica cross of rice (Oryza sativa L.). Breed. Sci. 52:319-325. doi:10.1270/jsbbs. 52.319

Lee, H.S., K. Sasaki, A. Higashitani, A. Sang-Nag, and S. Tadashi. 2012. Mapping and characterization of quantitative trait loci for mesocotyl elongation in rice (Oryza sativa L.). Rice 5:13. doi:10.1186/1939-8433-5-13

Lincoln, S.E., M.J. Daly, and E.S. Lander. 1993. Constructing genetic linkage maps with MAPMAKER/EXP Version 3.0: A tutorial and reference manual. Whitehead Institute, Cambridge Center, Cambridge, MA.

Liu, S., R. Zhou, Y.D.P. Li, and J. Jia. 2006. Development, utilization of introgression lines using a synthetic wheat as donor. Theor. Appl. Genet. 112:1360-1373. doi:10.1007/s00122-006-0238-x

Mace, E.S., H.K. Buhariwalla, and J.H. Crouch. 2003. A high throughput DNA extraction protocol for tropical molecular breeding programs. Plant Mol. Biol. Rep. 21:459a-459h. doi:10.1007/BF02772596

Money, T.A., C.J. Liu, and M.D. Gale. 1994. Conversion of RFLP markers for downy mildew resistance in pearl millet to sequence-tagged sites. In: J.R. Witcombe and R.R. Duncan, editors, Use of molecular markers in sorghum and pearl millet breeding for developing countries. Overseas Development Administration, London, UK. p. 65-68.
Nepolean, T., M. Blümmel, A.G. Bhasker Raj, V. Rajaram, S. Senthilvel, and C.T. Hash. 2006. QTLs controlling yield and stover quality traits in pearl millet. International Sorghum and Millets Newsletter. 47:149-152.

Qi, X., S. Lindup, T.S. Pittaway, S. Allouis, M.D. Gale, and K.M. Devos. 2001. Development of simple sequence repeat markers from bacterial artificial chromosomes without subcloning. Biotechniques 31:355-361.

Qi, X., T.S. Pittaway, S. Lindup, H. Liu, E. Waterman, F.K. Padi, C.T. Hash, J. Zhu, M.D. Gale, and K.M. Devos. 2004. An integrated genetic map of pearl millet, Pennisetum glaucum. Theor. Appl. Genet. 109:14851493. doi:10.1007/s00122-004-1765-y

Rai, K.N., C.T. Hash, A.K. Singh, and G. Velu. 2008. Adaptation and quality traits of a germplasm-derived commercial seed parent of pearl millet. Plant Genet. Resour. Newsl. 154:20-24.

Rajaram, V., T. Nepolean, S. Senthilvel, R.K. Varshney, V. Vadez, R.K. Srivastava, T. Shah, A. Supriya, S. Kumar, B.R. Kumari, A. Bhanuprakash, M.L. Narasu, O. Riera-Lizarazu, and C.T. Hash. 2013. Pearl millet [Pennisetum glaucum (L.) R. Br.] consensus linkage map constructed using four RIL mapping populations and newly developed EST-SSRs. BMC Genomics 14:159. doi:10.1186/1471-2164-14-159

Ramsay, L.D., D.E. Jennings, E.R. Bohuon, A.E. Arthur, D.J. Lydiate, M.J. Kearsey, and D.F. Marshall. 1996. The construction of a substitution library of recombinant backcross lines in Brassica oleracea for the precision mapping of quantitative trait loci. Genome 39:558-567. doi:10.1139/g96-071

Rooney, L.W., and C.M. McDonough. 1987. Food quality and consumer acceptance in pearl millet. In: J.R. Witcombe and S.R. Beckerman, editors, Proceedings of the International Pearl Millet Workshop. ICRISAT, Patancheru, Andhra Pradesh, India. 7-11 Apr. 1986. ICRISAT Center, India. p. 43-61.

Senthilvel, S., B. Jayashree, V. Mahalakshmi, P. Sathish Kumar, S. Nakka, T. Nepolean, and C.T. Hash. 2008. Development and mapping of simple sequence repeat markers for pearl millet from data mining of expressed sequence tags. BMC Plant Biol. 8:119. doi:10.1186/1471-2229-8-119

Singh, S.D., P. Singh, K.N. Rai, and D.J. Andrews. 1990. Registration of ICMA 841 and ICMB 841 pearl millet parental lines with A1 cytoplasmic-genic male sterility system. Crop Sci. 30:1378. doi:10.2135/cropsci1 990.0011183X003000060081x

Tegelstrom, H. 1992. Detection of mitochondrial DNA fragments. In: A.R. Hoelzel, editor, Molecular genetic analysis of populations: A practical approach. IRL Press, Oxford, UK. p. 89-114.

Tian, F., Z. Zhu, B. Zhang, L. Tan, Y. Fu, X. Wang, and C.Q. Sun. 2006. Fine mapping of a quantitative trait locus for grain number per panicle from wild rice (Oryza rufipogon Griff.). Theor. Appl. Genet. 113:619-629. doi:10.1007/s00122-006-0326-y

Xu, J., Q. Zhao, P. Du, Ch. Xu, B. Wang, Q. Feng, Q. Liu, S. Tang, M. Gu, B. Han, and G. Liang. 2010. Developing high throughput genotyped chromosome segment substitution lines based on population wholegenome re-sequencing in rice (Oryza sativa L.). BMC Genomics 11:656. doi:10.1186/1471-2164-11-656

Yadav, R.S., C.T. Hash, F.R. Bidinger, K.M. Devos, and C.J. Howarth. 2004. Genomic regions associated with grain yield and aspects of post-flowering drought tolerance in pearl millet across stress environments and tester background. Euphytica 136:265-277. doi:10.1023/ B:EUPH.0000032711.34599.3a

Yadav, R.S., C.T. Hash, G.P. Cavan, F.R. Bidinger, and C.J. Howarth. 2002. Quantitative trait loci associated with traits determining grain and stover yield in pearl millet under terminal drought stress conditions. Theor. Appl. Genet. 104:67-83. doi:10.1007/s001220200008

Yang, D., Y. Zhang, Z. Zhu, T. Chen, Q. Zhao, S. Yao, L. Zhao, W. Zhu, and C. Wang. 2013. Substitutional mapping the cooked rice elongation by using chromosome segment substitution lines in rice. Mol. Plant Breed. 4(13):107-115 doi:10.5376/mpb.2013.04.0013

Zhang, H., Q. Zhao, Z.Z. Sun, Ch.Q. Zhang, Q. Feng, S.H. Tang, G.H. Liang, G.H. Gu, B. Han, and Q.Q. Liu. 2011. Development and highthroughput genotyping of substitution lines carrying the chromosome segments of indica 9311 in the background of japonica Nipponbare. J. Genet. Genomics 38:603-611. doi:10.1016/j.jgg.2011.11.004 